\title{
Studies on stress- strain behaviour of concrete mixes confined with BFRP rebars
}

\author{
$K$ Ajay Kumar ${ }^{*}$, A Venkat Sai Krishna ${ }^{2}, S$ Shrihari ${ }^{3}$, and Srinivasa Reddy $V^{4}$ \\ ${ }^{1} \mathrm{M}$. Tech (Structural Engineering), Department of Civil Engineering, VJIT, Hyderabad, India. ${ }^{1}$ \\ ${ }^{2}$ Assistant Professor of Civil Engineering, VJIT, Hyderabad, India. \\ ${ }^{3}$ Professor of Civil Engineering, VJIT, Hyderabad, India. \\ ${ }^{4}$ Professor of Civil Engineering, GRIET, Hyderabad, India
}

\begin{abstract}
In the present study, the stress-stain behaviour of confined concrete made with basalt fibre reinforced polymer bars (BFRP) were taken up. The stress-strain behaviour was studied for the concrete mixes confined with steel rebars and BFRP rebars. The confinement was given in the form of steel hoops in the cylinders, 3 hoops $(0.8 \%)$, 4 hoops (1.1\%), 5 hoops $(1.3 \%)$ and 6 hoops $(1.6 \%)$. The addition of basalt fibres along with confinement of concrete with steel and BFRP hoops enhanced the compressive strength, indicating further confinement effect in the concrete. It is observed that the addition of fibres is helpful in lower confinements only. Beyond $1.1 \%$ confinement, the addition of any type of basalt fibres doesn't show any effect on compressive strengths. From the stress-strain behaviour of all types of concrete mixes, it is concluded that the ultimate load-carrying capacity and strains at peak stresses are more in concrete with BFRP hoops for mixes up to $1.1 \%$ confinement. The addition of basalt fibres to concrete has increased the ductility in both confined and unconfined states
\end{abstract}

\section{Introduction}

The Studies on Stress-Strain behaviour of concrete are essential in determining the parameters like energy absorption, toughness, plasticity index and they are very useful in design of structures using such concretes. Further modelling the stress-strain behaviour helps in predicating their behaviour. In the present study, the stress-stain behaviour of confined concrete made with basalt fibre reinforced polymer bars (BFRP) were done.

\section{Methodology}

In this phase of investigations, the stress-stain behaviour of concrete confined by steel and BFRP rebars were taken up. The confinement was given in the form of steel hoops in the cylinders, 3 hoops $(0.8 \%), 4$ hoops $(1.1 \%), 5$ hoops $(1.3 \%)$ and 6 hoops $(1.6 \%)$ as shown in Fig 1 . The tests were carried out on the standard cylindrical specimens of diameter $150 \mathrm{~mm}$ and height $300 \mathrm{~mm}$. After casting, the cylinders were capped with cement mortar and cured for a period of 28 days in curing tanks. The specimens were then taken out and made surface dry. The

\footnotetext{
* Corresponding author: ajaykandikonda03@gmail.com
}

samples were placed in a microprocessor strain controlled universal testing machine of $1000 \mathrm{kN}$ capacity and tested under uni-axial compression as per IS 516:1959. The stress-strain behaviour as obtained was plotted.

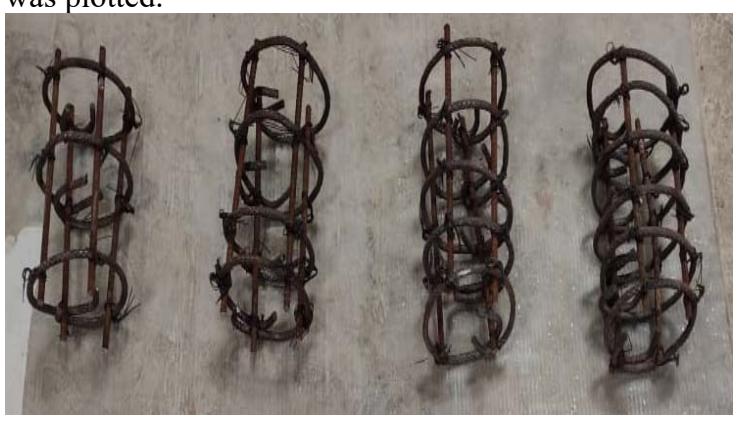

Fig.1. Cylinders with different Confinements 
Table 1. Percentage of confinement by volume

\begin{tabular}{|c|c|c|}
\hline & & Volume of confinement percentage \\
\hline C0 & Confinement 0 hoops & 0.00 \\
\hline C3 & Confinement 3 hoops & 0.80 \\
\hline C4 & Confinement 4 hoops & 1.1 \\
\hline C5 & Confinement 5 hoops & 1.3 \\
\hline C6 & Confinement 6 hoops & 1.6 \\
\hline
\end{tabular}

Table 2. Stress-strain values of concrete confined with steel bars

\begin{tabular}{|c|c|c|c|c|c|c|c|c|c|}
\hline \multicolumn{10}{|c|}{ CONFINED WITH STEEL BARS } \\
\hline M30-C0-STEEL & M30-C3-STEEL & M30-C4-STEEL & \multicolumn{1}{|c|}{ M30-C5-STEEL } & \multicolumn{2}{c|}{ M30-C6-STEEL } \\
\hline Strain & Stress & Strain & Stress & Strain & Stress & Strain & Stress & Strain & Stress \\
\hline 0.0000 & 0.00 & 0.0000 & 0.00 & 0.0000 & 0.00 & 0.0000 & 0.00 & 0.0000 & 0.00 \\
\hline 0.0000 & 0.17 & 0.0001 & 4.50 & 0.0000 & 0.66 & 0.0000 & 0.26 & 0.0002 & 8.61 \\
\hline 0.0000 & 0.34 & 0.0003 & 8.36 & 0.0001 & 4.80 & 0.0001 & 5.93 & 0.0004 & 14.09 \\
\hline 0.0001 & 4.48 & 0.0005 & 13.93 & 0.0002 & 8.95 & 0.0002 & 11.35 & 0.0005 & 19.57 \\
\hline 0.0003 & 8.97 & 0.0007 & 18.43 & 0.0003 & 12.66 & 0.0003 & 15.22 & 0.0006 & 23.22 \\
\hline 0.0005 & 12.59 & 0.0008 & 22.29 & 0.0005 & 18.56 & 0.0004 & 19.08 & 0.0008 & 30.26 \\
\hline 0.0007 & 16.72 & 0.0010 & 26.79 & 0.0006 & 22.93 & 0.0005 & 23.73 & 0.0010 & 34.96 \\
\hline 0.0008 & 20.52 & 0.0012 & 30.21 & 0.0007 & 26.42 & 0.0006 & 26.81 & 0.0011 & 38.61 \\
\hline 0.0010 & 23.62 & 0.0013 & 33.86 & 0.0008 & 31.00 & 0.0008 & 31.45 & 0.0013 & 42.52 \\
\hline 0.0012 & 27.24 & 0.0015 & 36.43 & 0.0010 & 34.72 & 0.0009 & 36.08 & 0.0015 & 45.39 \\
\hline 0.0013 & 29.48 & 0.0016 & 37.93 & 0.0011 & 37.55 & 0.0010 & 38.91 & 0.0016 & 48.00 \\
\hline 0.0016 & 34.48 & 0.0017 & 40.07 & 0.0012 & 39.30 & 0.0012 & 42.51 & 0.0017 & 49.30 \\
\hline 0.0017 & 36.90 & 0.0018 & 42.21 & 0.0014 & 44.54 & 0.0014 & 45.33 & 0.0019 & 51.13 \\
\hline 0.0019 & 36.90 & 0.0020 & 42.00 & 0.0015 & 44.76 & 0.0014 & 45.32 & 0.0020 & 52.17 \\
\hline 0.0021 & 34.66 & 0.0021 & 41.14 & 0.0016 & 43.01 & 0.0015 & 46.60 & 0.0022 & 50.61 \\
\hline 0.0022 & 31.38 & 0.0023 & 38.79 & 0.0017 & 40.39 & 0.0016 & 47.37 & 0.0022 & 48.78 \\
\hline 0.0023 & 28.28 & 0.0024 & 36.00 & 0.0018 & 37.55 & 0.0017 & 44.50 & 0.0024 & 45.13 \\
\hline 0.0025 & 25.86 & 0.0025 & 33.00 & 0.0019 & 35.59 & 0.0019 & 41.37 & 0.0025 & 41.74 \\
\hline 0.0025 & 24.14 & 0.0026 & 30.86 & 0.0019 & 34.06 & 0.0021 & 39.29 & 0.0026 & 37.57 \\
\hline 0.0026 & 22.07 & 0.0027 & 28.29 & & & 0.0022 & 37.45 & & \\
\hline
\end{tabular}




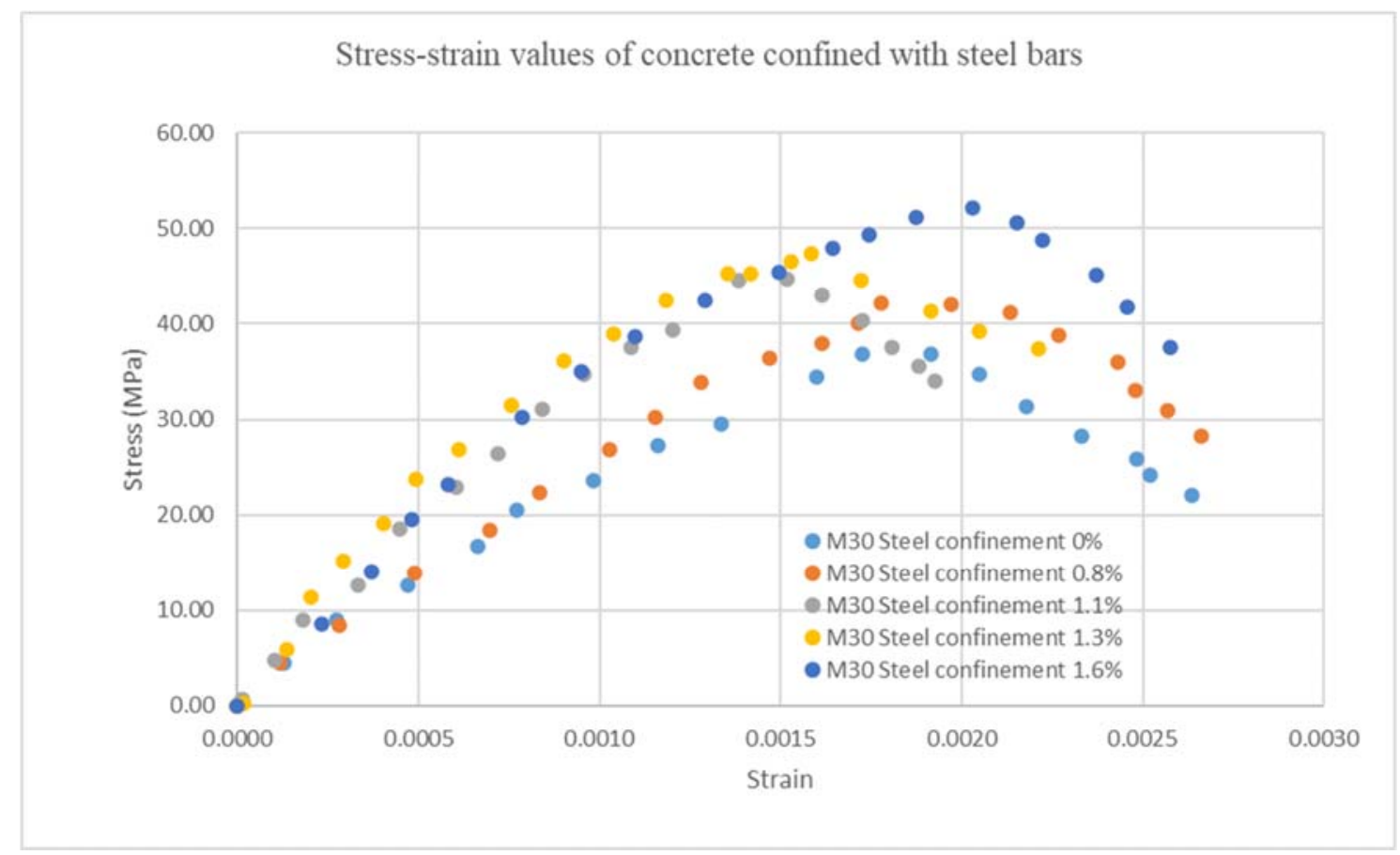

Fig. 1. Stress-strain values of concrete confined with steel bars

Table 3. Stress-strain values of concrete confined with BFRP bars

\begin{tabular}{|c|c|c|c|c|c|c|c|c|c|}
\hline \multicolumn{10}{|c|}{ CONFINED WITH BFRP BARS } \\
\hline M30-C0- BFRP & M30-C3- BFRP & \multicolumn{1}{c|}{ M30-C4- BFRP } & M30-C5- BFRP & \multicolumn{2}{c|}{ M30-C6- BFRP } \\
\hline 0.0000 & 0.00 & 0.0000 & 0.00 & 0.0000 & 0.00 & 0.0000 & 0.00 & 0.0000 & 0.00 \\
\hline 0.0001 & 8.66 & 0.0002 & 7.27 & 0.0002 & 6.90 & 0.0002 & 7.79 & 0.0000 & 0.87 \\
\hline 0.0003 & 13.85 & 0.0004 & 16.28 & 0.0004 & 19.47 & 0.0002 & 14.29 & 0.0001 & 7.42 \\
\hline 0.0005 & 19.74 & 0.0006 & 24.94 & 0.0008 & 27.63 & 0.0003 & 21.21 & 0.0002 & 13.52 \\
\hline 0.0006 & 25.97 & 0.0008 & 32.55 & 0.0011 & 39.71 & 0.0005 & 26.41 & 0.0003 & 20.49 \\
\hline 0.0007 & 29.44 & 0.0009 & 37.06 & 0.0015 & 50.93 & 0.0006 & 31.60 & 0.0005 & 29.21 \\
\hline 0.0009 & 34.63 & 0.0011 & 40.52 & 0.0018 & 62.14 & 0.0006 & 36.80 & 0.0006 & 37.05 \\
\hline 0.0011 & 41.90 & 0.0012 & 45.71 & 0.0021 & 68.15 & 0.0008 & 41.99 & 0.0006 & 41.42 \\
\hline 0.0014 & 46.75 & 0.0014 & 49.87 & 0.0025 & 74.14 & 0.0010 & 47.19 & 0.0008 & 45.79 \\
\hline 0.0015 & 52.29 & 0.0015 & 54.72 & 0.0026 & 76.71 & 0.0011 & 51.95 & 0.0009 & 54.51 \\
\hline 0.0016 & 54.72 & 0.0017 & 60.26 & 0.0028 & 80.13 & 0.0012 & 57.58 & 0.0010 & 58.87 \\
\hline 0.0018 & 59.57 & 0.0020 & 64.42 & 0.0030 & 82.28 & 0.0013 & 62.34 & 0.0011 & 62.80 \\
\hline 0.0020 & 64.42 & 0.0022 & 67.88 & 0.0031 & 84.41 & 0.0015 & 67.53 & 0.0013 & 68.92 \\
\hline 0.0021 & 64.76 & 0.0024 & 73.42 & 0.0033 & 83.93 & 0.0017 & 71.86 & 0.0014 & 72.85 \\
\hline 0.0022 & 68.57 & 0.0027 & 73.42 & 0.0035 & 79.54 & 0.0018 & 78.35 & 0.0016 & 77.66 \\
\hline 0.0024 & 74.46 & 0.0029 & 67.19 & 0.0037 & 72.97 & 0.0020 & 80.95 & 0.0017 & 82.03 \\
\hline 0.0028 & 69.61 & 0.0031 & 60.95 & 0.0038 & 65.53 & 0.0022 & 83.98 & 0.0020 & 86.87 \\
\hline 0.0028 & 65.11 & 0.0031 & 55.76 & & & 0.0024 & 85.71 & 0.0022 & 88.63 \\
\hline 0.0029 & 59.22 & 0.0032 & 49.52 & & & 0.0025 & 87.45 & 0.0023 & 90.82 \\
\hline 0.0030 & 54.03 & & & & & 0.0026 & 86.15 & 0.0025 & 93.90 \\
\hline 0.0033 & 51.95 & & & & & 0.0027 & 84.85 & 0.0026 & 95.66 \\
\hline & & & & & & 0.0029 & 83.98 & 0.0028 & 96.57 \\
\hline & & & & & & 0.0030 & 79.22 & 0.0029 & 90.50 \\
\hline & & & & & & 0.0031 & 72.29 & 0.0030 & 86.16 \\
\hline & & & & & & & 0.0030 & 78.77 \\
\hline
\end{tabular}




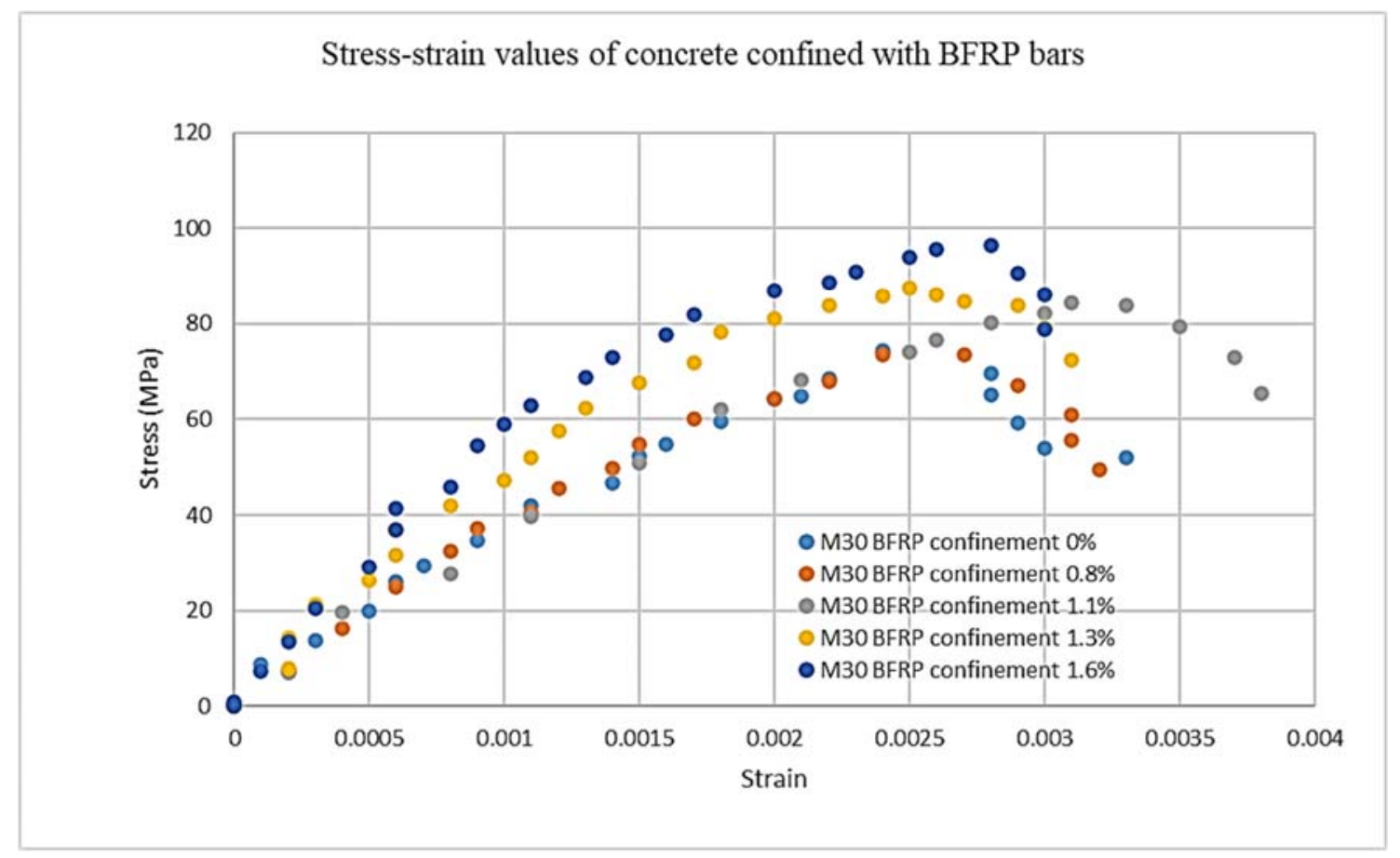

Fig. 2. Stress-strain values of concrete confined with steel bars

Table 4. Peak stresses of concrete confined with STEEL and BFRP bars

\begin{tabular}{|c|c|c|c|}
\hline \multirow{4}{*}{ Type } & Name & Strain at peak stress & $\begin{array}{c}\text { Peak stress } \\
\text { (MPa) }\end{array}$ \\
\hline \multirow{4}{*}{$\begin{array}{c}\text { NORMAL CONCRETE } \\
\text { CONFINED WITH STEEL }\end{array}$} & M30-C0-STEEL & 0.0019 & 36.90 \\
\cline { 2 - 4 } & M30-C3-STEEL & 0.0018 & 42.21 \\
\cline { 2 - 4 } & M30-C4-STEEL & 0.0015 & 44.76 \\
\cline { 2 - 4 } & M30-C5-STEEL & 0.0016 & 47.37 \\
\cline { 2 - 4 } & M30-C6-STEEL & 0.0020 & 52.17 \\
\hline \multirow{3}{*}{$\begin{array}{c}\text { BASALT FIBRE CONCRETE } \\
\text { CONFINED WITH BFRP BARS }\end{array}$} & M30-C0- BFRP & 0.0024 & 74.46 \\
\cline { 2 - 4 } & M30-C3- BFRP & 0.0027 & 73.42 \\
\cline { 2 - 4 } & M30-C4- BFRP & 0.0031 & 84.41 \\
\cline { 2 - 4 } & M30-C5- BFRP & 0.0025 & 87.45 \\
\cline { 2 - 4 } & M30-C6- BFRP & 0.0028 & 96.57 \\
\hline
\end{tabular}

\section{Conclusions}

1. The addition of fibres along with confinement of FRSCC with steel hoops enhanced the compressive strength, indicating further confinement effect in the FRSCC.

2. It is observed that the addition of fibres is helpful in lower confinements only. Beyond $1.1 \%$ confinement, the addition of any type of fibres doesn't show any effect on compressive strengths.

3. From the stress-strain behaviour of all types of FRSCC, it is concluded that the ultimate load-carrying capacity and strains at peak stresses are more in SFRSCC and HFRSCC for mixes up to $1.1 \%$ confinement.

4. The addition of fibres to SCC has increased the ductility in both confined and unconfined states.

\section{References}

1. T.Srinivas and M. Abinay Raj, Int. J. of Eng.and Adv. Tech. (IJEAT), ISSN: 2249 - 8958, Volume-8 Issue-6 (2019) 
2. T.srinivas and P. Manoj Anand, Int. J. of Innov. Tech. and Explor. Eng.g (IJITEE), ISSN: 22783075, Volume-8 Issue-12 (2019)

3. T.Srinivas and G. Sukesh Reddy, Int. J. of Eng.and Adv. Tech. (IJEAT), ISSN: 2249 8958, Volume-9 Issue-1 (2019)

4. T.Srinivas and R. N. Koushik, Int. J. of Innov. Tech. and Explor. Eng.g (IJITEE), ISSN: 22783075, Volume-8 Issue-12 (2019), PP 112-117.

5. K. Sai Gopi, Dr. T. Srinivas and S. P. Raju V, E3S Web of Conferences ICMED 184, 01084GRIET, 28-29 February, https://doi.org/10.1051/e3sconf/202018401108 4(2020)

6. Srinivas Rao J, S K Tummala, Kuthuri N R, Indonesia Journal of Electrical Engg. \& Computer Science, 21 (723), 2020

7. Jagannadha Kumar, M.V., Jagannadha Rao, K., Dean Kumar, B., Srinivasa Reddy, V., Int. J. of Civil Eng. and Tech., 9(7), pp. 1133-1141 (2018)

8. Ganta, J.K., Seshagiri Rao, M.V., Mousavi, S.S., Srinivasa Reddy, V., Bhojaraju, C., Structures 28, pp. 956-972 (2020)

9. Karthik Rao, R., Bobba, P.B., Suresh Kumar, T., Kosaraju, S. Materials Today: Proceedings, 26, pp. 3085-3089, 2019

10. Naidu, K.S.S.T., Rao, M.V.S., Reddy, V.S., Int. J. of Innov. Tech. and Explor. Eng.g (IJITEE), 8(9 Special Issue 2), pp. 641-642 (2019)

11. Chandana Priya, C., Seshagiri Rao, M.V., Srinivasa Reddy, V., Int. J. of Civil Eng. and Tech., 9(11), pp. 2218-2225 (2018)

12. R. Kumar, A. Sahoo, K. Satyanarayana, and G. Rao, International Journal of Industrial Engineering Computations, 4(3), 427 (2013)

13. Satya Sai Trimurty Naidu, K., Seshagiri Rao, M.V., Srinivasa Reddy, V., Int. J. of Civil Eng. and Tech., 9(11), pp. 2383-2393 (2018)

14. Supriya, Y., Srinivasa Reddy, V., Seshagiri Rao, M.V., Shrihari, S., Int. J. of Rec. Tech. and Engi., 8(3), pp. 5381-5385 (2019)

15. Kavitha, Merugu; Prasad, Dinkar; Bobba, Phaneendra Babu: IET Electric Power Applications, 2019, 13, (8), p. 1184-1192

16. Kotkunde, N., Krishna, G., Shenoy, S.K., Gupta, A.K., Singh, S.K. International Journal of Material Forming, 10 (2), pp. 255-266 (2017)

17. Govardhan, D., Kumar, A.C.S., Murti, K.G.K., Madhusudhan Reddy, G. Materials and Design, 36, pp. 206-214. (2012)

18. Kumar, P., Singhal, A., Mehta, S., Mittal, A. Journal of Real-Time Image Processing, 11 (1), pp. 93-109. (2016)

19. Raghunadha Reddy, T., Vishnu Vardhan, B., Vijayapal Reddy, P. International Journal of Applied Engineering Research, 11 (5), pp. 30923102 (2016)

20. Hussaini, S.M., Krishna, G., Gupta, A.K., Singh, S.K. Journal of Manufacturing Processes, 18, pp. 151-158 (2015) 